\title{
Spectral Analysis of $q$-Sturm-Liouville Problem with the Spectral Parameter in the Boundary Condition
}

\author{
Aytekin Eryılmaz \\ Department of Mathematics, Nevsehir University, Nevsehir 50300, Turkey \\ Correspondence should be addressed to Aytekin Ery1lmaz, eryilmazaytekin@gmail.com
}

Received 20 April 2012; Accepted 2 August 2012

Academic Editor: Feliz Minhós

Copyright (c) 2012 Aytekin Eryllmaz. This is an open access article distributed under the Creative Commons Attribution License, which permits unrestricted use, distribution, and reproduction in any medium, provided the original work is properly cited.

This paper is concerned with $q$-Sturm-Liouville boundary value problem in the Hilbert space with a spectral parameter in the boundary condition. We construct a self-adjoint dilation of the maximal dissipative $q$-difference operator and its incoming and outcoming spectral representations, which make it possible to determine the scattering matrix of the dilation. We prove theorems on the completeness of the system of eigenvalues and eigenvectors of operator generated by boundary value problem.

\section{Introduction}

Spectral analysis of Sturm-Liouville and Schrödinger differential equations with a spectral parameter in the boundary conditions has been analyzed intensively (see [1-16]). Then spectral analysis of discrete equations became an interesting subject in this field. So there is a substantial literature on this subject (see [10, 17-19]).

There has recently been great interest in quantum calculus and many works have been devoted to some problems of $q$-difference equation. In particular, we refer the reader to consult the reference [20] for some definitions and theorems on $q$-derivative, $q$-integration, $q$-exponential function, $q$-trigonometric function, $q$-Taylor formula, $q$-Beta and Gamma functions, Euler-Maclaurin formula, anf so forth. In [21], Ad $\imath$ var and Bohner investigated the eigenvalues and the spectral singularities of non-selfa-djoint $q$-difference equations of second order with spectral singularities. In [12], Huseynov and Bairamov examined the properties of eigenvalues and eigenvectors of a quadratic pencil of $q$-difference equations. In [22], Agarwal examined spectral analysis of self-adjoint equations. In [23], Shi and Wu presented several classes of explicit self-adjoint Sturm-Liouville difference operators with 
either a non-Hermitian leading coefficient function, or a non-Hermitian potential function, or a nondefinite weight function, or a non-self-adjoint boundary condition. In [24], Annaby and Mansour studied a $q$-analogue of Sturm-Liouville eigenvalue problems and formulated a self-adjoint $q$-difference operator in a Hilbert space. They also discussed properties of the eigenvalues and the eigenfunctions.

In this paper, we consider $q$-Sturm-Liouville Problem and define an adequate Hilbert space. Our main target of the present paper is to study $q$-Sturm-Liouville boundary value problem in case of dissipation at the right endpoint of $(0, a)$ and with the spectral parameter at zero. The maximal dissipative $q$-Sturm-Liouville operator is constructed using $[25,26]$ and Lax-Phillips scattering theory in [27]. Then we constructed a functional model of dissipative operator by means of the incoming and outcoming spectral representations and defined its characteristic function in terms of the solutions of the corresponding $q$-Sturm-Liouville equation. By combining the results of Nagy-Foiaş and Lax-Phillips, characteristic function is expressed with scattering matrix and the dilation of dissipative operator is set up. Finally, we give theorems on completeness of the system of eigenvectors and associated vectors of the dissipative $q$-difference operator.

Let $q$ be a positive number with $0<q<1, A \subset \mathbb{R}$, and $a \in \mathbb{C}$. A $q$-difference equation is an equation that contains $q$-derivatives of a function defined on $A$. Let $y(x)$ be a complexvalued function on $x \in A$. The $q$-difference operator $D_{q}$ is defined by

$$
D_{q} y(x)=\frac{y(q x)-y(x)}{\mu(x)}, \quad \forall x \in A,
$$

where $\mu(x)=(q-1) x$. The $q$-derivative at zero is defined by

$$
D_{q} y(0)=\lim _{n \rightarrow \infty} \frac{y\left(q^{n} x\right)-y(0)}{q^{n} x}, \quad x \in A,
$$

if the limit exists and does not depend on $x$. A right inverse to $D q$, the Jackson $q$-integration, is given by

$$
\int_{0}^{x} f(t) d_{q} t=x(1-q) \sum_{n=0}^{\infty} q^{n} f\left(q^{n} x\right), \quad x \in A,
$$

provided that the series converges, and

$$
\int_{a}^{b} f(t) d_{q} t=\int_{0}^{b} f(t) d_{q} t-\int_{0}^{a} f(t) d_{q} t, \quad a, b \in A .
$$

Let $L_{q}^{2}(0, a)$ be the space of all complex-valued functions defined on $[0, a]$ such that

$$
\|f\|:=\left(\int_{0}^{a}|f(x)| d_{q} x\right)^{1 / 2}<\infty
$$


Journal of Function Spaces and Applications

The space $L_{q}^{2}(0, a)$ is a separable Hilbert space with the inner product

$$
(f, g):=\int_{0}^{a} f(x) \overline{g(x)} d_{q} x, \quad f, g \in L_{q}^{2}(0, a)
$$

We will consider the basic Sturm-Liouville equation

$$
l(y):=-\frac{1}{q} D_{q^{-1}} D_{q} y(x)+v(x) y(x), \quad 0 \leq x \leq a<+\infty,
$$

where $v(x)$ is defined on $[0, a]$ and continuous at zero. The $q$-Wronskian of $y_{1}(x), y_{2}(x)$ is defined to be

$$
W_{q}\left(y_{1}, y_{2}\right)(x):=y_{1}(x) D_{q} y_{2}(x)-y_{2}(x) D_{q} y_{1}(x), \quad x \in[0, a]
$$

Let $L_{0}$ denote the closure of the minimal operator generated by (1.7) and by $D_{0}$ its domain. Besides, we denote by $D$ the set of all functions $y(x)$ from $L_{q}^{2}(0, a)$ such that $y(x)$ and $D_{q} y(x)$ are continuous in $[0, a)$ and $l(y) \in L_{q}^{2}(0, a) ; D$ is the domain of the maximal operator $L$. Furthermore, $L=L_{0}^{*}[2,4,13]$. Suppose that the operator $L_{0}$ has defect index $(2,2)$.

For every $y, z \in D$ we have $q$-Lagrange's identity [24]

$$
(L y, z)-(y, L z)=[y, \bar{z}](a)-[y, \bar{z}](0)
$$

where $[y, \bar{z}]:=y(x) \overline{D_{q^{-1}} z(x)}-D_{q^{-1}} y(x) \overline{z(x)}$

\section{Construction of the Dissipative Operator}

Consider boundary value problem governed by

$$
(l y)=\lambda y, \quad y \in D
$$

subject to the boundary conditions

$$
\begin{gathered}
y(a)-h D_{q^{-1}} y(a)=0, \quad \operatorname{Im} h>0, \\
\alpha_{1} y(0)-\alpha_{2} D_{q^{-1}} y(0)=\lambda\left(\alpha_{1}^{\prime} y(0)-\alpha_{2}^{\prime} D_{q^{-1}} y(0)\right),
\end{gathered}
$$

where $\lambda$ is spectral parameter and $\alpha_{1}, \alpha_{2}, \alpha_{1}^{\prime}, \alpha_{2}^{\prime} \in \mathbb{R}$ and $\alpha$ is defined by

$$
\alpha:=\left|\begin{array}{ll}
\alpha_{1}^{\prime} & \alpha_{1} \\
\alpha_{2}^{\prime} & \alpha_{2}
\end{array}\right|=\alpha_{1}^{\prime} \alpha_{2}-\alpha_{1} \alpha_{2}^{\prime}>0
$$


For convenience we assume

$$
\begin{gathered}
R_{0}(y):=\alpha_{1} y(0)-\alpha_{2} D_{q^{-1}} y(0), \\
R_{0}^{\prime}(y):=\alpha_{1}^{\prime} y(0)-\alpha_{2}^{\prime} D_{q^{-1}} y(0), \\
N_{1}^{a}(y):=y(a), \\
N_{2}^{a}(y):=D_{q^{-1}} y(a), \\
N_{1}^{0}(y):=y(0), \\
N_{2}^{0}(y):=D_{q^{-1}} y(0)_{\infty}, \\
R_{a}(y):=N_{2}^{a}(y)-h N_{1}^{a}(y) .
\end{gathered}
$$

Lemma 2.1. For arbitrary $y, z \in D$, let one suppose that $R_{0}(\bar{z})=\overline{R_{0}(z)}, R_{0}^{\prime}(\bar{z})=\overline{R_{0}^{\prime}(z)}$, then one has the following.

Proof.

$$
\begin{gathered}
{[y, z]_{0}=\frac{1}{\alpha}\left[R_{0}(y) \overline{R_{0}^{\prime}(z)}-R_{0}^{\prime}(y) \overline{R_{0}(z)}\right],} \\
\frac{1}{\alpha}\left[R_{0}(y) R_{0}^{\prime} \overline{(z)}-R_{0}^{\prime}(y) \overline{R_{0}(z)}\right] \\
=\frac{1}{\alpha}\left[\begin{array}{c}
\left(\alpha_{1} y(0)-\alpha_{2} D_{q^{-1}} y(0)\right)\left(\overline{\alpha_{1}^{\prime} z(0)-\alpha_{2}^{\prime} D_{q^{-1}} z(0)}\right) \\
-\left(\alpha_{1}^{\prime} y(0)-\alpha_{2}^{\prime} D_{q^{-1}} y(0)\right)\left(\overline{\alpha_{1} z(0)-\alpha_{2} D_{q^{-1}} z(0)}\right)
\end{array}\right] \\
=\frac{1}{\alpha}\left[\left(\alpha_{1}^{\prime} \alpha_{2}-\alpha_{1} \alpha_{2}^{\prime}\right)\left(\overline{y(0)} D_{q^{-1}} z(0)-D_{q^{-1}} y(0) \overline{z(0)}\right)\right] \\
=[y, z]_{0} .
\end{gathered}
$$

Let $\theta_{1}, \theta_{2}$ denote the solutions of (2.1) satisfying the conditions

$$
N_{1}^{0}\left(\theta_{2}\right)=\alpha_{2}-\alpha_{2}^{\prime} \lambda, \quad N_{2}^{0}\left(\theta_{2}\right)=\alpha_{1}-\alpha_{1}^{\prime} \lambda, \quad N_{1}^{a}\left(\theta_{1}\right)=h, \quad N_{2}^{a}\left(\theta_{1}\right)=1
$$

Then from (2.3) we have

$$
\begin{aligned}
\Delta(\lambda) & =\left[\theta_{1}, \theta_{2}\right]_{x}=-\left[\theta_{2}, \theta_{1}\right]_{x}=-\left[\theta_{2}, \theta_{1}\right]_{0} \\
& =-\frac{1}{\alpha}\left[R_{0}\left(\theta_{1}\right) \overline{R_{0}^{\prime}\left(\theta_{2}\right)}-R_{0}^{\prime}\left(\theta_{1}\right) \overline{R_{0}\left(\theta_{2}\right)}\right] \\
& =R_{0}\left(\theta_{2}\right)-\lambda R_{0}^{\prime}\left(\theta_{2}\right),
\end{aligned}
$$


Journal of Function Spaces and Applications

$$
\begin{aligned}
\Delta(\lambda) & =\left[\theta_{1}, \theta_{2}\right]_{x}=-\left[\theta_{2}, \theta_{1}\right]_{x}=-\left[\theta_{2}, \theta_{1}\right]_{a} \\
& =-\left(y(a) \overline{D_{q^{-1}} z(a)}-z(a) \overline{D_{q^{-1}} y(a)}\right) \\
& =-\left(y(a)-h \overline{D_{q^{-1}} y(a)}\right)=-\left(N_{2}^{a}\left(\theta_{1}\right)-h N_{1}^{a}\left(\theta_{1}\right)\right) .
\end{aligned}
$$

We let

$$
G(x, \xi, \lambda)=\frac{-1}{\Delta(\lambda)}\left\{\begin{array}{ll}
\theta_{2}(\xi, \lambda) \theta_{1}(x, \lambda), & x<\xi \\
\theta_{1}(x, \lambda) \theta_{2}(\xi, \lambda), & \xi<x
\end{array}\right\}
$$

It can be shown that $G(x, \xi, \lambda)$ satisfies (2.1) and boundary conditions (2.2)-(2.3). $G(x, \xi, \lambda)$ is a Green function of the boundary value problem (2.1)-(2.3). Thus, we obtain that the $G(x, \xi, \lambda)$ is a Hilbert-Schmidt kernel and the solution of the boundary value problem can be expressed by

$$
y(x, \lambda)=\int_{0}^{a} G(x, \xi, \lambda) y(\xi, \lambda) d \xi=R_{\curlywedge} y
$$

Thus $R_{\lambda}$ is a Hilbert Schmidt operator on space $L_{q}^{2}(0, a)$. The spectrum of the boundary value problem coincides with the roots of the equation $\Delta(\lambda)=0$. Since $\Delta$ is analytic and not identical to zero, it means that the function $\Delta$ has at most a countable number of isolated zeros with finite multiplicity and possible limit points at infinity.

Suppose that $f^{(1)} \in L_{2}[0, a), f^{(2)} \in \mathbb{C}$, then we denote linear space $H=L_{q}^{2}(0, a) \oplus \mathbb{C}$ with two component of elements of $\widehat{f}=\left(\begin{array}{c}f^{(1)} \\ f^{(2)}\end{array}\right)$. If $\alpha>0$ and $\widehat{f}=\left(\begin{array}{c}f^{(1)} \\ f^{(2)}\end{array}\right), \widehat{g}=\left(\begin{array}{l}g^{(1)} \\ g^{(2)}\end{array}\right) \in H$, then the formula

$$
(\widehat{f}, \widehat{g})=\int_{0}^{a} f^{(1)}(x) \bar{g}^{(1)} d_{q} x+\frac{1}{\alpha} f^{(2)} \bar{g}^{(2)}
$$

defines an inner product in Hilbert space $H$. Let us define operator of $A_{h}: H \rightarrow H$ with equalities suitable for boundary value problem

$$
\begin{gathered}
D\left(A_{h}\right)=\left\{\widehat{f}=\left(\begin{array}{c}
f^{(1)} \\
f^{(2)}
\end{array}\right) \in H: f^{(1)} \in D, R_{a}\left(f^{(1)}\right)=0, f^{(2)}=R_{0}^{\prime}\left(f^{(1)}\right)\right\}, \\
A_{h} \widehat{f}=\tilde{l}(\widehat{f}):=\left(\begin{array}{c}
l\left(f^{(1)}\right) \\
R_{0}\left(f^{(1)}\right)
\end{array}\right) .
\end{gathered}
$$

Remind that a linear operator $A_{h}$ with domain $D\left(A_{h}\right)$ in Hilbert space $H$ is called dissipative if $\operatorname{Im}\left(A_{h} f, f\right) \geq 0$ for all $f \in D\left(A_{h}\right)$ and maximal dissipative if it does not have a proper extension. 
Definition 2.2. If the system of vectors of $y_{0}, y_{1}, y_{2}, \ldots, y_{n}$ corresponding to the eigenvalue $\lambda_{0}$ is

$$
\begin{gathered}
l\left(y_{0}\right)=\lambda_{0} y_{0}, \quad R_{0}\left(y_{0}\right)-\lambda R_{0}^{\prime}\left(y_{0}\right)=0, \quad R_{a}\left(y_{0}\right)=0, \\
l\left(y_{s}\right)-\lambda_{0} y_{s}-y_{s-1}=0, \quad R_{0}\left(y_{s}\right)-\lambda R_{0}^{\prime}\left(y_{s}\right)-R_{0}^{\prime}\left(y_{s-1}\right)=0, \\
R_{a}\left(y_{s}\right)=0, \quad s=1,2, \ldots, n,
\end{gathered}
$$

then the system of vectors of $y_{0}, y_{1}, y_{2}, \ldots, y_{n}$ corresponding to the eigenvalue $\lambda_{0}$ is called a chain of eigenvectors and associated vectors of boundary value problem (2.2)-(2.12).

Since the operator $A_{h}$ is dissipative in $H$ and from Definition 2.2, we have the following.

Lemma 2.3. The eigenvalue of boundary value problem (2.1)-(2.3) coincides with the eigenvalue of dissipative $A_{h}$ operator. Additionally each chain of eigenvectors and associated vectors $y_{0}, y_{1}$, $y_{2}, \ldots, y_{n}$ corresponding to the eigenvalue $\lambda_{0}$ corresponds to the chain eigenvectors and associated vectors $\widehat{y}_{0}, \widehat{y}_{1}, \widehat{y}_{2}, \ldots, \widehat{y}_{n}$ corresponding to the same eigenvalue $\lambda_{0}$ of dissipative $A_{h}$ operator. In this case, the equality

$$
\widehat{y}_{k}=\left(\begin{array}{c}
y_{k} \\
R_{0}^{\prime}\left(y_{k}\right)
\end{array}\right), \quad k=0,1,2, \ldots, n
$$

holds.

Proof. $\hat{y}_{0} \in D\left(A_{h}\right)$ and $A_{h} \widehat{y}_{0}=\lambda_{0} \hat{y}_{0}$, then the equality $l\left(y_{0}\right)=\lambda_{0} y_{0}, R_{0}\left(y_{0}\right)-\lambda R_{0}^{\prime}\left(y_{0}\right)=0$, $R_{1}\left(y_{0}\right)=R_{2}\left(y_{0}\right)=0$ takes place; that is, $y_{0}$ is an eigenfunction of the problem. Conversely, if conditions (2.14) are realized, then $\left(\begin{array}{c}y_{0} \\ R_{0}^{\prime}\left(y_{0}\right)\end{array}\right)=\widehat{y}_{0} \in D\left(A_{h}\right)$ and $A_{h} \widehat{y}_{0}=\lambda_{0} \widehat{y}_{0}, \widehat{y}_{0}$ is an eigenvector of the operator $A_{h}$. If $\widehat{y}_{0}, \widehat{y}_{1}, \widehat{y}_{2}, \ldots, \widehat{y}_{n}$ are a chain of the eigenvectors and associated vectors of the operator $A_{h}$ corresponding to the eigenvalue $\lambda_{0}$, then by implementing the conditions $\widehat{y}_{k} \in D\left(A_{h}\right)(k=0,1,2, \ldots, n)$ and equality $A_{h} \widehat{y}_{0}=\lambda_{0} \widehat{y}_{0}, A_{h} \widehat{y}_{s}=$ $\lambda_{0} \widehat{y}_{s}+\widehat{y}_{s-1}, s=1,2, \ldots, n$, we get the equality (2.15), where $y_{0}, y_{1}, y_{2}, \ldots, y_{n}$ are the first components of the vectors $\widehat{y}_{0}, \widehat{y}_{1}, \widehat{y}_{2}, \ldots, \widehat{y}_{n}$. On the contrary, on the basis of the elements $y_{0}, y_{1}, y_{2}, \ldots, y_{n}$ corresponding to $(2.1)-(2.3)$, one can construct the vectors $\widehat{y}_{k}=\left(\begin{array}{c}y_{k} \\ R_{0}^{\prime}\left(y_{k}\right)\end{array}\right)$ for which $\widehat{y}_{k} \in D\left(A_{h}\right)(k=0,1,2, \ldots, n)$ and $A_{h} \widehat{y}_{0}=\lambda_{0} \widehat{y}_{0}, A_{h} \widehat{y}_{s}=\lambda_{0} \widehat{y}_{s}+\widehat{y}_{s-1}, s=1,2, \ldots, n$.

Theorem 2.4. The operator $A_{h}$ is maximal dissipative in the space $H$.

Proof. Let $\widehat{y} \in D\left(A_{h}\right)$. From (2.6), we have

$$
\begin{aligned}
\left(A_{h} \widehat{y}, \widehat{y}\right)-\left(\widehat{y}, A_{h} \widehat{y}\right) & =\left[y_{1}, y_{1}\right]_{a}-\left[y_{1}, \overline{y_{1}}\right]_{0}+\frac{1}{\alpha}\left[R_{0}\left(y_{1}\right) R_{0}^{\prime} \overline{\left(y_{1}\right)}-R_{0}^{\prime}\left(y_{1}\right) \overline{R_{0}\left(y_{1}\right)}\right] \\
& =\left[y_{1}, y_{1}\right]_{a}=2 \operatorname{Im} h\left(D_{q^{-1}} y_{1}(a)\right)^{2}
\end{aligned}
$$


It follows from that $\operatorname{Im}\left(A_{h} \widehat{y}, \widehat{y}\right)=\operatorname{Im} h\left(D_{q^{-1}} y_{1}(a)\right)^{2} \geq 0, A_{h}$ is a dissipative operator in $H$. Let us prove that $A_{h}$ is maximal dissipative operator in the space $H$. It is sufficient to check that

$$
\left(A_{h}-\lambda I\right) D\left(A_{h}\right)=H, \quad \operatorname{Im} \lambda<0 .
$$

To prove (2.17), let $F \in H, \operatorname{Im} \lambda<0$ and put

$$
\Gamma=\left(\begin{array}{c}
\left(\widetilde{G}_{x}, \bar{F}\right) \\
R_{0}^{\prime}\left[\left(\widetilde{G}_{x}, \bar{F}\right)\right]
\end{array}\right)
$$

where

$$
\begin{aligned}
& \tilde{G}_{x}=\left(\begin{array}{c}
G(x, \xi, \lambda) \\
R_{0}^{\prime}[G(x, \xi, \lambda)]
\end{array}\right)=\left(\begin{array}{c}
G(x, \xi, \lambda) \\
\frac{-1}{\Delta(\lambda)} \theta_{1}(x, \lambda) \alpha
\end{array}\right), \\
& G(x, \xi, \lambda)=\frac{-1}{\Delta(\lambda)}\left\{\begin{array}{ll}
\theta_{2}(\xi, \lambda) \theta_{1}(x, \lambda), & x<\xi \\
\theta_{1}(x, \lambda) \theta_{2}(\xi, \lambda), & \xi<x
\end{array}\right\} .
\end{aligned}
$$

The function $x \rightarrow\left(G(x, \xi, \lambda), \overline{F_{1}}\right)$ satisfies the equation $l(y)-\lambda y=F_{1}(0 \leq x<\infty)$ and the boundary conditions (2.1)-(2.3). Moreover, for all $F \in H$ and for $\operatorname{Im} \lambda<0$, we arrive at $\Gamma \in D\left(A_{h}\right)$. For each $F \in H$ and for $\operatorname{Im} \lambda<0$, we have $\left(A_{h}-\lambda I\right) \Gamma=F$. Consequently, in the case of $\operatorname{Im} \lambda<0$, the result is $\left(A_{h}-\lambda I\right) D\left(A_{h}\right)=H$. Hence, Theorem 2.4 is proved.

\section{Self-Adjoint Dilation of Dissipative Operator}

We first construct the self-adjoint dilation of the operator $A_{h}$. Let us add the "incoming" and "outgoing" subspaces $D_{-}=L^{2}(-\infty, 0]$ and $D_{+}=L^{2}[0, \infty)$ to $H=L_{q}^{2}(0, a) \oplus \mathbb{C}$. The orthogonal sum $\mathrm{H}=D_{-} \oplus H \oplus D_{+}$is called main Hilbert space of the dilation. In the space $\mathscr{d}$ we consider the operator $\mathcal{L}_{h}$ on the set $D\left(\mathcal{L}_{h}\right)$, its elements consisting of vectors $w=\left\langle\varphi_{-}, y, \varphi_{+}\right\rangle$, generated by the expression

$$
\mathcal{L}\left\langle\varphi_{-}, \widehat{y}, \varphi_{+}\right\rangle=\left\langle i \frac{d \varphi_{-}}{d \xi}, \tilde{l}(\widehat{y}), i \frac{d \varphi_{+}}{d \xi}\right\rangle
$$

satisfying the conditions: $\varphi_{-} \in W_{2}^{1}(-\infty, 0], \varphi_{+} \in W_{2}^{1}[0, \infty), \widehat{y} \in H, \widehat{y}=\left(\begin{array}{c}y_{1}(x) \\ y_{2}\end{array}\right), y_{1} \in D, y_{2}=$ $R_{0}\left(y_{1}\right)$, and

$$
y(a)-h D_{q^{-1}} y(a)=\beta \varphi_{-}(0), \quad y(a)-\bar{h} D_{q^{-1}} y(a)=\beta \varphi_{+}(0)
$$

where $W_{2}^{1}(\cdot, \cdot)$ are Sobolev spaces and $\beta^{2}:=2 \operatorname{Im} h, \beta>0$. Then we have the following.

Theorem 3.1. The operator $\mathcal{L}_{h}$ is self-adjoint in $\mathcal{L}$ and it is a self-adjoint dilation of the operator $A_{h}$. 
Proof. We first prove that $\mathcal{L}_{h}$ is symmetric in $\mathscr{d}$. Namely $\left(\mathcal{L}_{h} f, g\right)_{\mathscr{d}}-\left(f, \mathcal{L}_{h} g\right)_{\mathscr{d}}=0$. Let $f, g \in$ $D\left(\mathcal{L}_{h}\right), f=\left\langle\varphi_{-}, \hat{y}, \varphi_{+}\right\rangle$and $g=\left\langle\psi_{-}, \widehat{z}, \psi_{+}\right\rangle$. Then we have

$$
\begin{aligned}
&\left(\mathcal{L}_{h} f, g\right)_{\mathscr{H}}-\left(f, \mathcal{L}_{h} g\right)_{\mathscr{H}}=\left(\mathcal{L}\left\langle\varphi_{-}, \widehat{y}, \varphi_{+}\right\rangle,\left\langle\psi_{-}, \widehat{z}, \psi_{+}\right\rangle\right)-\left(\left\langle\varphi_{-}, \widehat{y}, \varphi_{+}\right\rangle, \mathcal{L}\left\langle\psi_{-}, \widehat{z}, \psi_{+}\right\rangle\right), \\
&= {\left[y_{1}, \overline{z_{1}}\right]_{a}-\left[y_{1}, \overline{z_{1}}\right]_{0}+\frac{1}{\alpha}\left[R_{0}\left(y_{1}\right) \overline{R_{0}^{\prime}\left(z_{1}\right)}-R_{0}^{\prime}\left(y_{1}\right) \overline{R_{0}\left(z_{1}\right)}\right] } \\
&+i \psi_{-}(0) \bar{\varphi}_{-}(0)-i \varphi_{+}(0) \bar{\psi}_{+}(0), \\
&\left(\mathcal{L}_{h} f, g\right)_{\mathscr{H}}-\left(f, \mathcal{L}_{h} g\right)_{\mathscr{H}}=\left[y_{1}, \bar{z}_{1}\right]_{a}+i \psi_{-}(0) \bar{\varphi}_{-}(0)-i \varphi_{+}(0) \bar{\psi}_{+}(0) .
\end{aligned}
$$

On the other hand,

$$
\begin{aligned}
i \psi_{-}(0) \bar{\varphi}_{-}(0)-i \varphi_{+}(0) \bar{\psi}_{+}(0)= & \frac{i}{\beta^{2}}\left(y(a)-h D_{q^{-1}} y(a)\right) \overline{\left(z(a)-h D_{q^{-1}} z(a)\right)} \\
& -\frac{i}{\beta^{2}}\left(y(a)-\bar{h} D_{q^{-1}} y(a)\right) \overline{\left(z(a)-\bar{h} D_{q^{-1}} z(a)\right)}, \\
= & \frac{i}{\beta^{2}}\left[(h-\bar{h}) y(a) \overline{D_{q^{-1}} z(a)}-D_{q^{-1}} y(a) \overline{z(a)}\right]
\end{aligned}
$$

By (3.3), we have

$$
i \psi_{-}(0) \bar{\varphi}_{-}(0)-i \varphi_{+}(0) \bar{\psi}_{+}(0)=-\left[y_{1}, \overline{z_{1}}\right]_{a} .
$$

From equalities (3.3) and (3.5), we have $\left(\mathcal{L}_{h} f, g\right)_{\mathscr{d}}-\left(f, \mathcal{L}_{h} g\right)_{\mathscr{d}}=0$. Thus, $\mathcal{L}_{h}$ is a symmetric operator. To prove that $\mathcal{L}_{h}$ is self-adjoint, we need to show that $\mathcal{L}_{h} \subseteq \mathcal{L}_{h}^{*}$. We consider the bilinear form $\left(\mathcal{L}_{h} f, g\right)_{\mathscr{H}}$ on elements $g=\left\langle\psi_{-}, \widehat{z}, \psi_{+}\right\rangle \in D\left(\mathcal{L}_{h}^{*}\right)$, where $f=\left\langle\varphi_{-}, \widehat{y}, \varphi_{+}\right\rangle \in D\left(\mathcal{L}_{h}\right)$, $\varphi_{\mp} \in W_{2}^{1}\left(\mathbb{R}_{\mp}\right), \varphi_{\mp}(0)=0$. Integrating by parts, we get $\mathcal{L}_{h}^{*} g=\left\langle i\left(d \psi_{-} / d \xi\right), \hat{z}^{*}, i\left(d \psi_{+} / d \xi\right)\right\rangle$, where $\psi_{\mp} \in W_{2}^{1}\left(\mathbb{R}_{\mp}\right), \widehat{z}^{*} \in H$. Similarly, if $f=\langle 0, \widehat{y}, 0\rangle \in D\left(\mathcal{L}_{h}\right)$, then integrating by parts in $\left(\mathcal{L}_{h} f, g\right)_{\mathscr{L}}$, we obtain

$$
\mathcal{L}_{h}^{*} g=\mathcal{L}^{*}\left\langle\psi_{-}, \widehat{z}, \psi_{+}\right\rangle=\left\langle i \frac{d \psi_{-}}{d \xi}, \tilde{l}(\widehat{z}), i \frac{d \psi_{+}}{d \xi}\right\rangle, \quad z_{1} \in D, z_{2}=R_{0}^{\prime}\left(z_{1}\right)
$$

Consequently, we have $\left(\mathcal{L}_{h} f, g\right)_{\mathscr{\ell}}=\left(f, \mathcal{L}_{h} g\right)_{\mathscr{H}}$, for each $f \in D\left(\mathcal{L}_{h}\right)$ by (3.6), where the operator $\mathcal{L}$ is defined by (3.1). Therefore, the sum of the integrated terms in the bilinear form $\left(\mathcal{L}_{h} f, g\right)_{\mathscr{\ell}}$ must be equal to zero:

$$
\left[y_{1}, \overline{z_{1}}\right]_{a}-\left[y_{1}, \overline{z_{1}}\right]_{0}+\frac{1}{\alpha}\left[R_{0}\left(y_{1}\right) \overline{R_{0}^{\prime}\left(z_{1}\right)}-R_{0}^{\prime}\left(y_{1}\right) \overline{R_{0}\left(z_{1}\right)}\right]+i \varphi_{-}(0)^{\prime} \bar{\psi}_{-}(0)-i \varphi_{+}^{\prime}(0) \bar{\psi}_{+}(0)=0 .
$$

Then by (2.6), we get

$$
\left[y_{1}, \bar{z}_{1}\right]_{a}+i \varphi_{-}(0)^{\prime} \bar{\psi}_{-}(0)-i \varphi_{+}^{\prime}(0) \bar{\psi}_{+}(0)=0
$$


From the boundary conditions for $\mathcal{L}_{h}$, we have

$$
\begin{gathered}
y(a)=\beta \varphi_{-}(0)-\frac{h_{1}}{i \beta}\left(\varphi_{-}(0)-\varphi_{+}(0)\right), \\
D_{q^{-1}} y(a)=\frac{i}{\beta}\left(\varphi_{-}(0)-\varphi_{+}(0)\right) .
\end{gathered}
$$

Afterwards, by (3.8) we get

$$
\begin{aligned}
\beta \varphi_{-}(0) & -\frac{h_{1}}{i \beta}\left(\varphi_{-}(0)-\varphi_{+}(0)\right) \overline{z(a)}-\frac{i}{\beta}\left(\varphi_{-}(0)-\varphi_{+}(0)\right) \overline{D_{q^{-1}} z(a)} \\
= & i \varphi_{+}(0) \bar{\psi}_{+}(0)-i \varphi_{-}(0) \bar{\psi}_{-}(0) .
\end{aligned}
$$

Comparing the coefficients of $\varphi_{-}(0)$ in (3.10), we obtain

$$
\frac{i \beta^{2}-h_{1}}{\beta} z(a)+\frac{1}{\beta} \overline{D_{q^{-1}} z(a)}=\varphi_{-}(0)
$$

or

$$
z(a)-h D_{q^{-1}} z(a)=\beta \psi_{-}(0)
$$

Similarly, comparing the coefficients of $\varphi_{+}(0)$ in (3.10) we get

$$
z(a)-\bar{h} D_{q^{-1}} z(a)=\beta \psi_{+}(0)
$$

Therefore conditions (3.12) and (3.13) imply $D\left(\mathcal{L}_{h}^{*}\right) \subseteq D\left(\mathcal{L}_{h}\right)$, hence $\mathcal{L}_{h}=\mathcal{L}_{h}^{*}$.

The self-adjoint operator $\mathcal{L}_{h}$ generates on $\mathscr{L}$ a unitary group $U_{t}=\exp \left(i \mathcal{L}_{h} t\right)\left(t \in \mathbb{R}_{+}=\right.$ $(0, \infty))$. Let us denote by $P: \mathscr{H} \rightarrow H$ and $P_{1}: H \rightarrow \mathscr{H}$ the mapping acting according to the formulae $P:\left\langle\varphi_{-}, \widehat{y}, \varphi_{+}\right\rangle \rightarrow \widehat{y}$ and $P_{1}: \hat{y} \rightarrow\langle 0, \widehat{y}, 0\rangle$. Let $Z_{t}:=P U_{t} P_{1}, t \geq 0$, by using $U_{t}$. The family $\left\{Z_{t}\right\}(t \geq 0)$ of operators is a strongly continuous semigroup of completely nonunitary contraction on $H$. Let us denote by $B_{h}$ the generator of this semigroup: $B_{h} \widehat{y}=\lim _{t \rightarrow+0}(i t)^{-1}$ $\left(Z_{t} \hat{y}-\widehat{y}\right)$. The domain of $B_{h}$ consists of all the vectors for which the limit exists. The operator $B_{h}$ is dissipative. The operator $\mathcal{L}_{h}$ is called the self-adjoint dilation of $B_{h}$ (see $[2,9,18]$ ). We show that $B_{h}=A_{h}$, hence $\mathcal{L}_{h}$ is self-adjoint dilation of $B_{h}$. To show this, it is sufficient to verify the equality

$$
P\left(\mathcal{L}_{h}-\lambda I\right)^{-1} P_{1} \widehat{y}=\left(A_{h}-\lambda I\right)^{-1} \widehat{y}, \quad \widehat{y} \in H, \operatorname{Im} h<0 .
$$

For this purpose, we set $\left(\mathcal{L}_{h}-\lambda I\right)^{-1} P_{1} \hat{y}=g=\left\langle\psi_{-}, \widehat{z}, \psi_{+}\right\rangle$which implies that $\left(\mathcal{L}_{h}-\lambda I\right) g=P_{1} \hat{y}$, and hence $\widetilde{l}(\widehat{z})-\lambda \widehat{z}=\widehat{y}, \psi_{-}(\xi)=\psi_{-}(0) e^{-i \lambda \xi}$ and $\psi_{+}(\xi)=\psi_{+}(0) e^{-i \lambda \xi}$. Since $g \in D\left(\mathcal{L}_{h}\right)$, then $\psi_{-} \in L^{2}(-\infty, 0)$, and it follows that $\psi_{-}(0)=0$, and consequently $z$ satisfies the boundary condition $z(a)-h D_{q^{-1}} z(a)=0$. Therefore, $\widehat{z} \in D\left(A_{h}\right)$, and since point $\lambda$ with $\operatorname{Im} \lambda<0$ cannot 
be an eigenvalue of dissipative operator, it follows that $\psi_{+}(0)$ is obtained from the formula $\psi_{+}(0)=\beta^{-1}\left(z(a)-\bar{h} D_{q^{-1}} z(a)\right)$. Thus

$$
\left(\mathcal{L}_{h}-\lambda I\right)^{-1} P_{1} \widehat{y}=\left\langle 0,\left(A_{h}-\lambda I\right)^{-1} \widehat{y}, \beta^{-1}\left(z(a)-\bar{h} D_{q^{-1}} z(a)\right)\right\rangle
$$

for $\widehat{y}$ and $\operatorname{Im} \lambda<0$. On applying the mapping $P$, we obtain (3.14), and

$$
\begin{aligned}
\left(A_{h}-\lambda I\right)^{-1} & =P\left(\mathcal{L}_{h}-\lambda I\right)^{-1} P_{1}=-i P \int_{0}^{\infty} U_{t} e^{-i \lambda t} d t P_{1} \\
& =-i \int^{\infty} Z_{t} e^{-i \lambda t} d t=\left(B_{h}-\lambda I\right)^{-1}, \quad \operatorname{Im} \lambda<0,
\end{aligned}
$$

so this clearly shows that $A_{h}=B_{h}$.

The unitary group $\left\{U_{t}\right\}$ has an important property which makes it possible to apply it to the Lax-Phillips [27], that is, it has orthogonal incoming and outcoming subspaces $D_{-}=$ $\left\langle L^{2}(-\infty, 0), 0,0\right\rangle$ and $D_{+}=\left\langle 0,0, L^{2}(0, \infty)\right\rangle$ having the following properties:

(1) $U_{t} D_{-} \subset D_{-}, t \leq 0$ and $U_{t} D_{+} \subset D_{+}, t \geq 0$;

(2) $\cap_{t \leq 0} U_{t} D_{-}=\cap_{t \geq 0} U_{t} D_{+}=\{0\}$;

(3) $\cup_{t \geq 0} U_{t} D_{-}=\cup_{t \leq 0} U_{t} D_{+}=\mathscr{H}$;

(4) $D_{-} \perp D_{+}$.

To be able to prove property (1) for $D_{+}$(the proof for $D_{-}$is similar), we set $R_{\lambda}=$ $\left(\mathcal{L}_{h}-\lambda I\right)^{-1}$. For all $\lambda$, with $\operatorname{Im} \lambda<0$ and for any $f=\left\langle 0,0, \varphi_{+}\right\rangle \in D_{+}$, we have

$$
\mathcal{R}_{\lambda} f=\left\langle 0,0,-i e^{-i \lambda \xi} \int_{0}^{\xi} e^{i \lambda s} \varphi_{+}(s) d s\right\rangle,
$$

as $\mathcal{R}_{\lambda} f \in D_{+}$. Therefore, if $g \perp D_{+}$, then

$$
0=\left(\mathcal{R}_{\lambda} f, g\right)_{\mathscr{d}}=-i \int_{0}^{\infty} e^{-i \lambda t}\left(U_{t} f, g\right)_{\mathscr{H}} d t, \quad \operatorname{Im} \lambda<0
$$

which implies that $\left(U_{t} f, g\right)_{\mathscr{\ell}}=0$ for all $t \geq 0$. Hence, for $t \geq 0, U_{t} D_{+} \subset D_{+}$, and property (1) has been proved.

In order to prove property (2), we define the mappings $P^{+}: \mathscr{d} \rightarrow L^{2}(0, \infty)$ and $P_{1}^{+}$: $L^{2}(0, \infty) \rightarrow D_{+}$as follows: $P^{+}:\left\langle\varphi_{-}, \widehat{y}, \varphi_{+}\right\rangle \rightarrow \varphi_{+}$and $P_{1}^{+}: \varphi \rightarrow\langle 0,0, \varphi\rangle$, respectively. We take into consideration that the semigroup of isometries $U_{t}^{+}:=P^{+} U_{t} P_{1}^{+}(t \geq 0)$ is a one-sided shift in $L^{2}(0, \infty)$. Indeed, the generator of the semigroup of the one-sided shift $V_{t}$ in $L^{2}(0, \infty)$ is the differential operator $i(d / d \xi)$ with the boundary condition $\varphi(0)=0$. On the other hand, the generator $S$ of the semigroup of isometries $U_{t}^{+}(t \geq 0)$ is the operator $S \varphi=P^{+} \mathcal{L}_{h} P_{1}^{+} \varphi=$ 
$P^{+} \mathcal{L}_{h}\langle 0,0, \varphi\rangle=P^{+}\langle 0,0, i(d / d \xi) \varphi\rangle=i(d / d \xi) \varphi$, where $\varphi \in W_{2}^{1}(0, \infty)$ and $\varphi(0)=0$. Since a semigroup is uniquely determined by its generator, it follows that $U_{t}^{+}=V_{t}$, and hence

$$
\bigcap_{t \geq 0} U_{t} D_{+}=\left\langle 0,0, \bigcap_{t \leq 0} V_{t} L^{2}(0, \infty)\right\rangle=\{0\},
$$

so, the proof of property (2) is completed.

Definition 3.2. The linear operator $A$ with domain $D(A)$ acting in the Hilbert space $H$ is called completely non-self-adjoint (or simple) if there is no invariant subspace $M \subseteq D(A)(M \neq\{0\})$ of the operator $A$ on which the restriction $A$ to $M$ is self-adjoint.

To prove property (3) of the incoming and outcoming subspaces, let us prove following lemma.

Lemma 3.3. The operator $A_{h}$ is completely non-self-adjoint (simple).

Proof. Let $H^{\prime} \subset H$ be a nontrivial subspace in which $A_{h}$ induces a self-adjoin t operator $A_{h}^{\prime}$ with domain $D\left(A_{h}^{\prime}\right)=H^{\prime} \cap D\left(A_{h}\right)$. If $\widehat{f} \in D\left(A_{h}^{\prime}\right)$, then $\widehat{f} \in D\left(A_{h}^{\prime *}\right)$ and

$$
\begin{aligned}
\frac{d}{d t}\left\|e^{i A_{h}^{\prime} t} \widehat{f}\right\|_{H}^{2} & =\frac{d}{d t}\left(e^{i A_{h}^{\prime} t} \widehat{f}, e^{i A_{h}^{\prime} t} \widehat{f}\right)_{H} \\
& =i\left(A_{h}^{\prime} e^{i A_{h}^{\prime} t} \widehat{f}, e^{i A_{h}^{\prime} t} \widehat{f}\right)-i\left(e^{i A_{h}^{\prime} t} \widehat{f}, A_{h}^{\prime} e^{i A_{h}^{\prime} t} \widehat{f}\right)
\end{aligned}
$$

and taking $\widehat{g}=e^{i A_{h}^{\prime} t} \widehat{f}$, we have

$$
\begin{aligned}
0 & =i\left(A_{h}^{\prime} \widehat{g}, \widehat{g}\right)_{H}-i\left(\widehat{g}, A_{h}^{\prime} \widehat{g}\right)_{H} \\
& =i\left[g_{1}, \overline{g_{1}}\right]_{a}-i\left[g_{1}, \overline{g_{1}}\right]_{0}+\frac{i}{\alpha}\left[R_{0}\left(g_{1}\right) \overline{R_{0}^{\prime}\left(g_{1}\right)}-R_{0}^{\prime}\left(y_{1}\right) \overline{R_{0}\left(g_{1}\right)}\right] \\
& =-2 \operatorname{Im} h\left(D_{q^{-1}} y_{1}(a)\right)^{2} \\
& =-\beta^{2}\left(D_{q^{-1}} y_{1}(a)\right)^{2} .
\end{aligned}
$$

Since $\widehat{f} \in D\left(A_{h}^{\prime}\right), A_{h}^{\prime}$ holds condition above. Moreover, eigenvectors of the operator $A_{h}^{\prime}$ should also hold this condition. Therefore, for the eigenvectors $\widehat{y}(\lambda)$ of the operator $A_{h}$ acting in $H^{\prime}$ and the eigenvectors of the operator $A_{h^{\prime}}^{\prime}$ we have $D_{q^{-1}} y_{1}(a)=0$. From the boundary conditions, we get $y_{1}(a)=0$ and $\widehat{y}(x, \lambda)=0$. Consequently, by the theorem on expansion in the eigenvectors of the self-adjoint operator $A_{h^{\prime}}^{\prime}$, we obtain $H^{\prime}=\{0\}$. Hence the operator $A_{h}$ is simple. The proof is completed.

Let us define $H_{-}=\overline{U_{t \geq 0} U_{t} D_{-}}, H_{+}=\overline{U_{t \leq 0} U_{t} D_{+}}$.

Lemma 3.4. The equality $H_{-}+H_{+}=\mathscr{d}$ holds. 
Proof. Considering property (1) of the subspace $D_{+}$, it is easy to show that the subspace $\mathscr{\ell}^{\prime}=$ $\mathscr{H} \odot\left(H_{-}+H_{+}\right)$is invariant relative to the group $\left\{U_{t}\right\}$ and has the form $\mathscr{\ell}^{\prime}=\left\langle 0, H^{\prime}, 0\right\rangle$, where $H^{\prime}$ is a subspace in $H$. Therefore, if the subspace $\mathscr{H}^{\prime}$ (and hence also $H^{\prime}$ ) was nontrivial, then the unitary group $\left\{U_{t}\right\}$ restricted to this subspace would be a unitary part of the group $\left\{U_{t}^{\prime}\right\}$, and hence the restriction $B_{h}^{\prime}$ of $B_{h}$ to $H^{\prime}$ would be a self-adjoint operator in $H^{\prime}$. Since the operator $B_{h}$ is simple, it follows that $H^{\prime}=\{0\}$. The lemma is proved.

Assume that $\varphi(\lambda)$ and $\psi(\lambda)$ are solutions of $l(y)=\lambda y$ satisfying the conditions

$$
\begin{gathered}
\varphi_{1}(0, \lambda)=0, \quad \varphi_{2}(0, \lambda)=1, \quad \psi_{1}(0, \lambda)=1, \quad \psi_{2}(0, \lambda)=0 . \\
\theta(x, \lambda)=\varphi(x, \lambda)+m_{a}(\lambda) \psi(x, \lambda) \in L_{q}^{2}(0, a), \quad \operatorname{Im} \lambda>0 .
\end{gathered}
$$

The Titchmarsh-Weyl function $m_{a}(\lambda)$ is a meromorphic function on the complex plane $\mathbb{C}$ with a countable number of poles on the real axis. Further, it is possible to show that the function $m_{a}(\lambda)$ possesses the following properties: $\operatorname{Im} m_{a}(\lambda) \geq 0$ for all $\operatorname{Im} \lambda>0$, and $\overline{m_{a}(\lambda)}=m_{a}(\bar{\lambda})$ for all $\lambda \in \mathbb{C}$, except the real poles $m_{a}(\lambda)$. We set

$$
\begin{gathered}
S_{h}(\lambda):=\frac{m_{a}(\lambda)-h}{m_{a}(\lambda)-\bar{h}} \\
U_{\lambda}^{-}(x, \xi, \zeta)=\left\langle e^{-i \lambda \xi},\left(m_{a}(\lambda)-h\right)^{-1} \alpha \theta(x, \lambda), \overline{S_{h}}(\lambda) e^{-i \lambda \zeta}\right\rangle .
\end{gathered}
$$

We note that the vectors $U_{\lambda}^{-}(x, \xi, \zeta)$ for real $\lambda$ do not belong to the space $\mathcal{H}$. However, $U_{\lambda}^{-}(x, \xi, \zeta)$ satisfies the equation $\mathcal{L} U=\lambda U$ and the corresponding boundary conditions for the operator $\mathcal{L}_{H}$. By means of vector $U_{\lambda}^{-}(x, \xi, \zeta)$, we define the transformation $F_{-}: f \rightarrow \tilde{f}_{-}(\lambda)$ by

$$
(F-f)(\lambda):=\tilde{f}_{-}(\lambda):=\frac{1}{\sqrt{2 \pi}}\left(f, U_{\bar{\lambda}}\right)_{\mathscr{\ell}^{\prime}}
$$

on the vectors $f=\left\langle\varphi_{-}, \hat{y}, \varphi_{+}\right\rangle$in which $\varphi_{-}(\xi), \varphi_{+}(\zeta), y(x)$ are smooth, compactly supported functions.

Lemma 3.5. The transformation $F_{-}$isometrically maps $H_{-}$onto $L^{2}(\mathbb{R})$. For all vectors $f, g \in H_{-}$the Parseval equality and the inversion formulae hold:

$$
(f, g)_{d \ell}=\left(\tilde{f}_{-}, \tilde{g}_{-}\right)_{L^{2}}=\int_{-\infty}^{\infty} \tilde{f}_{-}(\lambda) \overline{\widetilde{g}_{-}(\lambda)} d \lambda, \quad f=\frac{1}{\sqrt{2 \pi}} \int_{-\infty}^{\infty} \tilde{f}_{-}(\lambda) U_{\bar{\lambda}} d \lambda,
$$

where $\tilde{f}_{-}(\lambda)=\left(F_{-} f\right)(\lambda)$ and $\tilde{g}_{-}(\lambda)=\left(F_{-} g\right)(\lambda)$.

Proof. For $f, g \in D_{-}, f=\left\langle\varphi_{-}, 0,0\right\rangle, g=\left\langle\psi_{+}, 0,0\right\rangle$, with Paley-Wiener theorem, we have

$$
\tilde{f}_{-}(\lambda)=\frac{1}{\sqrt{2 \pi}}\left(f, U_{\bar{\lambda}}\right)_{\mathscr{d}}=\frac{1}{\sqrt{2 \pi}} \int_{-\infty}^{0} \varphi_{-}(\xi) e^{-i \lambda \xi} d \xi \in H_{-}^{2}
$$


and by using usual Parseval equality for Fourier integrals

$$
(f, g)_{d}=\int_{-\infty}^{\infty} \varphi_{-}(\xi) \overline{\psi_{-}(\xi)} d \xi=\int_{-\infty}^{\infty} \tilde{f}_{-}(\lambda) \overline{\tilde{g}_{-}(\lambda)} d \lambda=\left(F_{-} f, F_{-} g\right)_{L^{2}} .
$$

Here, $H_{ \pm}^{2}$ denote the Hardy classes in $L^{2}(\mathbb{R})$ consisting of the functions analytically extendible to the upper and lower half-planes, respectively.

We now extend to the Parseval equality to the whole of $H_{-}$. We consider in $H_{-}$the dense set of $H_{-}^{\prime}$ of the vectors obtained as follows from the smooth, compactly supported functions in $D_{-}: f \in H_{-}^{\prime}$ if $f=U_{T} f_{0}, f_{0}=\left\langle\varphi_{-}, 0,0\right\rangle, \varphi_{-} \in C_{0}^{\infty}(-\infty, 0)$, where $T=T_{f}$ is a nonnegative number depending on $f$. If $f, g \in H_{-}^{\prime}$, then for $T>T_{f}$ and $T>T_{g}$ we have $U_{-T} f, U_{-T} g \in D_{-} ;$moreover, the first components of these vectors belong to $C_{0}^{\infty}(-\infty, 0)$. Therefore, since the operators $U_{t}(t \in \mathbb{R})$ are unitary, by the equality

$$
F_{-} U_{t} f=\left(U_{t} f, U_{\bar{\lambda}}\right)_{\mathscr{d}}=e^{i \lambda t}\left(f, U_{\lambda}^{-}\right)_{\mathscr{d}}=e^{i \lambda t} F_{-} f,
$$

we have

$$
\begin{gathered}
(f, g)_{\mathscr{H}}=\left(U_{-T} f, U_{-T} g\right)_{\mathscr{H}}=\left(F_{-} U_{-T} f, F_{-} U_{-T} g\right)_{L^{2}} \\
\left(e^{i \lambda T} F_{-} f, e^{i \lambda T} F_{-} g\right)_{L^{2}}=(\tilde{f}, \tilde{g})_{L^{2}} .
\end{gathered}
$$

By taking the closure (3.30), we obtain the Parseval equality for the space $H_{-}$. The inversion formula is obtained from the Parseval equality if all integrals in it are considered as limits in the of integrals over finite intervals. Finally $F_{-} H_{-}=\overline{U_{t \geq 0} F_{-} U_{t} D_{-}}=\overline{U_{t \geq 0} e^{i \lambda t} H_{-}^{2}}=L^{2}(\mathbb{R})$, that is, $F_{-}$maps $H_{-}$onto the whole of $L^{2}(\mathbb{R})$. The lemma is proved.

We set

$$
U_{\lambda}^{+}(x, \xi, \zeta)=\left\langle S_{h}(\lambda) e^{-i \lambda \xi},\left(m_{a}(\lambda)-\bar{h}\right)^{-1} \alpha \theta(x, \lambda), e^{-i \lambda \zeta}\right\rangle .
$$

We note that the vectors $U_{\lambda}^{+}(x, \xi, \zeta)$ for real $\lambda$ do not belong to the space $\mathscr{H}$. However,

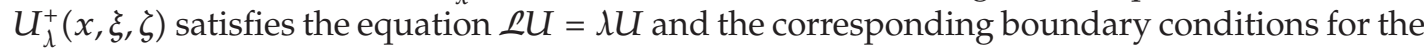
operator $\mathcal{L}_{H}$. With the help of vector $U_{\lambda}^{+}(x, \xi, \zeta)$, we define the transformation $F_{+}: f \rightarrow \tilde{f}_{+}(\lambda)$ by $\left(F_{+} f\right)(\lambda):=\tilde{f}_{+}(\lambda):=(1 / \sqrt{2 \pi})\left(f, U_{\lambda}^{+}\right)_{\mathscr{\ell}}$ on the vectors $f=\left\langle\varphi_{-}, \hat{y}, \varphi_{+}\right\rangle$in which $\varphi_{-}(\xi), \varphi_{+}(\zeta)$ and $y(x)$ are smooth, compactly supported functions.

Lemma 3.6. The transformation $F_{+}$isometrically maps $H_{+}$onto $L^{2}(\mathbb{R})$. For all vectors $f, g \in H_{+}$the Parseval equality and the inversion formula hold:

$$
(f, g)_{\mathscr{d}}=\left(\tilde{f}_{+}, \tilde{g}_{+}\right)_{L^{2}}=\int_{-\infty}^{\infty} \tilde{f}_{+}(\lambda) \overline{\widetilde{g}_{+}(\lambda)} d \lambda, \quad f=\frac{1}{\sqrt{2 \pi}} \int_{-\infty}^{\infty} \tilde{f}_{+}(\lambda) U_{\lambda}^{+} d \lambda,
$$

where $\tilde{f}_{+}(\lambda)=\left(F_{+} f\right)(\lambda)$ and $\tilde{g}_{+}(\lambda)=\left(F_{+} g\right)(\lambda)$. 
Proof. The proof is analogous to Lemma 3.5.

It is obvious that the matrix-valued function $S_{h}(\lambda)$ is meromorphic in $\mathbb{C}$ and all poles are in the lower half-plane. From (3.23), $\left|S_{h}(\lambda)\right| \leq 1$ for $\operatorname{Im} \lambda>0$; and $S_{h}(\lambda)$ is the unitary matrix for all $\lambda \in \mathbb{R}$. Therefore, it explicitly follows from the formulae for the vectors $U_{\lambda}^{-}$and $U_{\lambda}^{+}$that

$$
U_{\lambda}^{+}=S_{h}(\lambda) U_{\lambda}^{-}
$$

It follows from Lemmas 3.5 and 3.6 that $H_{-}=H_{+}$. Together with Lemma 3.4, this shows that $H_{-}=H_{+}=\mathscr{l}$; therefore, property (3) above has been proved for the incoming and outcoming subspaces. Finally property (4) is clear.

Thus, the transformation $F_{-}$isometrically maps $H_{-}$onto $L^{2}(\mathbb{R})$ with the subspace $D_{-}$ mapped onto $H_{-}^{2}$ and the operators $U_{t}$ are transformed into the operators of multiplication by $e^{i \lambda t}$. This means that $F_{-}$is the incoming spectral representation for the group $\left\{U_{t}\right\}$. Similarly, $F_{+}$is the outgoing spectral representation for the group $\left\{U_{t}\right\}$. It follows from (3.33) that the passage from the $F_{-}$representation of an element $f \in$ th to its $F_{+}$representation is accomplished as $\tilde{f}_{+}(\lambda)=S_{h}^{-1}(\lambda) \tilde{f}_{-}(\lambda)$. Consequently, according to [27] we have proved the following.

Theorem 3.7. The function $S_{h}^{-1}(\lambda)$ is the scattering matrix of the group $\left\{U_{t}\right\}$ (of the selfadjoint operator $L_{H}$ ).

Let $S(\lambda)$ be an arbitrary nonconstant inner function on the upper half-plane (the analytic function $S(\lambda)$ on the upper half-plane $\mathbb{C}_{+}$is called inner function on $\mathbb{C}_{+}$if $\left|S_{h}(\lambda)\right| \leq 1$ for all $\lambda \in \mathbb{C}_{+}$and $\left|S_{h}(\lambda)\right|=1$ for almost all $\left.\lambda \in \mathbb{R}\right)$. Define $K=H_{+}^{2} \ominus S H_{+}^{2}$. Then $K \neq\{0\}$ is a subspace of the Hilbert space $H_{+}^{2}$. We consider the semigroup of operators $Z_{t}(t \geq 0)$ acting in $K$ according to the formula $Z_{t} \varphi=P\left[e^{i \lambda t} \varphi\right], \varphi=\varphi(\lambda) \in K$, where $P$ is the orthogonal projection from $H_{+}^{2}$ onto $K$. The generator of the semigroup $\left\{Z_{t}\right\}$ is denoted by

$$
T \varphi=\lim _{t \rightarrow+0}(i t)^{-1}\left(Z_{t} \varphi-\varphi\right),
$$

in which $T$ is a maximal dissipative operator acting in $K$ and with the domain $D(T)$ consisting of all functions $\varphi \in K$, such that the limit exists. The operator $T$ is called a model dissipative operator (we remark that this model dissipative operator, which is associated with the names of Lax-Phillips [27], is a special case of a more general model dissipative operator constructed by Nagy and Foiaş [26]). The basic assertion is that $S(\lambda)$ is the characteristic function of the operator $T$.

Let $K=\langle 0, H, 0\rangle$, so that $\mathscr{L}=D_{-} \oplus K \oplus D_{+}$. It follows from the explicit form of the unitary transformation $F_{-}$under the mapping $F_{-}$

$$
\begin{gathered}
\mathscr{H} \longrightarrow L^{2}(\mathbb{R}), \quad f \longrightarrow \tilde{f}_{-}(\lambda)=\left(F_{-} f\right)(\lambda), \quad D_{-} \longrightarrow H_{-\prime}^{2} \quad D_{+} \longrightarrow S_{h} H_{+}^{2} \\
K \longrightarrow H_{+}^{2} \ominus S_{G} H_{+}^{2}, \quad U_{t} f \longrightarrow\left(F_{-} U_{t} F_{-}^{-1} \tilde{f}_{-}\right)(\lambda)=e^{i \lambda t} \tilde{f}_{-}(\lambda) .
\end{gathered}
$$

The formulae (3.35) show that operator $A_{h}$ is a unitarily equivalent to the model dissipative operator with the characteristic function $S_{h}(\lambda)$. Since the characteristic functions of unitary 
equivalent dissipative operator coincide (see [26]), we have thus proved the following theorem.

Theorem 3.8. The characteristic function of the maximal dissipative operator $A_{h}$ coincides with the function $S_{h}(\lambda)$ defined in (3.23).

Using characteristic function, the spectral properties of the maximal dissipative operator $A_{h}$ can be investigated. The characteristic function of the maximal dissipative operator $A_{h}$ is known to lead to information of completeness about the spectral properties of this operator. For instance, the absence of a singular factor $s(\lambda)$ of the characteristic function $S_{h}(\lambda)$ in the factorization det $S_{h}(\lambda)=s(\lambda) B(\lambda)$, where $B(\lambda)$ is a Blaschke product, ensures completeness of the system of eigenvectors and associated vectors of the operator $A_{h}$ in the space $L_{q}^{2}(0, a)$ (see [25]).

Theorem 3.9. For all the values of $h$ with $\operatorname{Im} h>0$, except possibly for a single value $h=h_{0}$, the characteristic function $S_{h}(\lambda)$ of the maximal dissipative operator $A_{h}$ is a Blaschke product. The spectrum of $A_{h}$ is purely discrete and belongs to the open upper half-plane. The operator $A_{h}$ has a countable number of isolated eigenvalues with finite multiplicity and limit points at infinity. The system of all eigenvectors and associated vectors of the operator $A_{h}$ is complete in the space $H$.

Proof. From (3.23), it is clear that $S_{h}(\lambda)$ is an inner function in the upper half-plane, and it is meromorphic in the whole complex $\lambda$-plane. Therefore, it can be factored in the form

$$
S_{h}(\lambda)=e^{i \lambda c} B_{h}(\lambda), \quad c=c(h) \geq 0,
$$

where $B_{h}(\lambda)$ is a Blaschke product. It follows from (3.36) that

$$
\left|S_{h}(\lambda)\right|=\left|e^{i \lambda c}\right|\left|B_{h}(\lambda)\right| \leq e^{-b(h) \operatorname{Im} \lambda}, \quad \operatorname{Im} \lambda \geq 0 .
$$

Further, for $m_{a}(\lambda)$ in terms of $S_{h}(\lambda)$, we find from (3.23) that

$$
m_{a}(\lambda)=\frac{h-\bar{h} S_{h}(\lambda)}{S_{h}(\lambda)-1}
$$

If $c(h)>0$ for a given value $h(\operatorname{Im} h>0)$, then (3.37) implies that $\lim _{t \rightarrow+\infty} S_{h}(i t)=0$, and then (3.24) gives us that $\lim _{t \rightarrow+\infty} m_{a}(i t)=-G$. Since $m_{a}(\lambda)$ does not depend on $h$, this implies that $c(h)$ can be nonzero at not more than a single point $h=h_{0}\left(\right.$ and further $\left.h_{0}=-\lim _{t \rightarrow+\infty} m_{a}(i t)\right)$. The theorem is proved.

Due to Theorem 2.4, since the eigenvalues of the boundary value problem (2.1)-(2.3) and eigenvalues of the operator $A_{h}$ coincide, including their multiplicity and, furthermore, for the eigenfunctions and associated functions the boundary problems (2.1)-(2.3), then theorem is interpreted as follows.

Corollary 3.10. The spectrum of the boundary value problem (2.1)-(2.3) is purely discrete and belongs to the open upper half-plane. For all the values of $h$ with $\operatorname{Im} \lambda>0$, except possible for a single value $h=h_{0}$, the boundary value problem (2.1)-(2.3) $\left(h \neq h_{0}\right)$ has a countable number of isolated 
eigenvalues with finite multiplicity and limit points and infinity. The system of the eigenfunctions and associated functions of this problem $\left(h \neq h_{0}\right)$ is complete in the space $L_{q}^{2}(0, a)$.

\section{References}

[1] B. P. Allakhverdiev and G. Sh. Guseřnov, “On the spectral theory of second-order dissipative difference operators," Matematicheskiน Sbornik, vol. 66, no. 1, pp. 107-125, 1990.

[2] B. P. Allahverdiev, "Dissipative second-order difference operators with general boundary conditions," Journal of Difference Equations and Applications, vol. 10, no. 1, pp. 1-16, 2004.

[3] B. P. Allahverdiev, "A nonself-adjoint singular Sturm-Liouville problem with a spectral parameter in the boundary condition," Mathematische Nachrichten, vol. 278, no. 7-8, pp. 743-755, 2005.

[4] B. P. Allahverdiev, "Extensions, dilations and functional models of infinite Jacobi matrix," Czechoslovak Mathematical Journal, vol. 55, no. 3, pp. 593-609, 2005.

[5] B. P. Allahverdiev, "A dissipative singular Sturm-Liouville problem with a spectral parameter in the boundary condition," Journal of Mathematical Analysis and Applications, vol. 20, pp. 75-92, 2006.

[6] F. V. Atkinson, Discrete and Continuous Boundary Problems, Academic Press, New York, NY, USA, 1964.

[7] J. Behrndt, M. M. Malamud, and H. Neidhardt, "Scattering theory for open quantum systems with finite rank coupling," Mathematical Physics, Analysis and Geometry, vol. 10, no. 4, pp. 313-358, 2007.

[8] C. T. Fulton, "Two-point boundary value problems with eigenvalue parameter contained in the boundary conditions," Proceedings of the Royal Society of Edinburgh A, vol. 77, no. 3-4, pp. 293-308, 1977.

[9] C. T. Fulton, "Singular eigenvalue problems with eigenvalue parameter contained in the boundary conditions," Proceedings of the Royal Society of Edinburgh A, vol. 87, no. 1-2, pp. 1-34, 1980.

[10] I. C. Gohberg and M. G. Krě̆n, Introduction to the Theory of Linear Nonselfadjoint Operators, American Mathematical Society, Providence, RI, USA, 1969.

[11] M. L. Gorbachuk and V. I. Gorbachuk, Granichnye Zadachi dlya Differentsialno-Operatornykh Uravnenii, Naukova Dumka, Kyiv, Ukrania, 1984, English translation, Birkhauser, 1991.

[12] A. Huseynov and E. Bairamov, "An eigenvalue problemd for quadratic pencils of $q$-difference equations and its applications," Applied Mathematics Letters, vol. 22, pp. 1-7, 2008.

[13] M. A. Nămark, Lineinye Differentsialnye Operatory, Nauka, Moscow, Russia, 2nd edition, 1969, English translation of 1st ed, vol. 1-2, Ungar, New York, NY, USA, 1967, 1968.

[14] M. Y. Ongun and B. P. Allahverdiev, "A completeness theorem for a dissipative Schrödinger problem with the spectral parameter in the boundary condition," Mathematische Nachrichten, vol. 281, no. 4, pp. 541-554, 2008.

[15] B. S. Pavlov, "Selfadjoint dilation of a dissipative Schrödinger operator and its resolution in terms of eigenfunctions," Mathematics of the USSR-Sbornik, vol. 31, no. 4, pp. 457-478, 1977.

[16] A. A. Shkalikov, "Boundary value problems with a spectral parameter in the boundary conditions," Zeitschrift für Angewandte Mathematik und Mechanik, vol. 76, pp. 133-135, 1996.

[17] E. Bairamov, O. Çakar, and A. M. Krall, "Non-selfadjoint difference operators and Jacobi matrices with spectral singularities," Mathematische Nachrichten, vol. 229, pp. 5-14, 2001.

[18] S. L. Clark, "A spectral analysis for self-adjoint operators generated by a class of second order difference equations," Journal of Mathematical Analysis and Applications, vol. 197, no. 1, pp. 267-285, 1996.

[19] S. T. Welstead, "Boundary conditions at infinity for difference equations of limit-circle type," Journal of Mathematical Analysis and Applications, vol. 89, no. 2, pp. 442-461, 1982.

[20] V. Kac and P. Cheung, Quantum Calculus, Springer, New York, NY, USA, 2002.

[21] M. Adıvar and M. Bohner, "Spectral analysis of $q$-difference equations with spectral singularities," Mathematical and Computer Modelling, vol. 43, no. 7-8, pp. 695-703, 2006.

[22] R. P. Agarwal, Difference Equations and Inequalities: Theory, Methods and Applications, Marcel Dekker, New York, NY, USA, 2nd edition, 2000.

[23] G. Shi and H. Wu, "Spectral theory of Sturm-Liouville difference operators," Linear Algebra and its Applications, vol. 430, no. 2-3, pp. 830-846, 2009.

[24] M. H. Annaby and Z. S. Mansour, "Basic Sturm-Liouville problems," Journal of Physics A, vol. 38, no. 17, pp. 3775-3797, 2005. 
[25] A. Kuzhel, Characteristic Functions and Models of Nonselfadjoint Operators, vol. 349, Kluwer Academic, Dordrecht, The Netherlands, 1996.

[26] Nagy, B. Sz, and C. Foiaş, Analyse Harmonique des Operateurs de L'espace de Hilbert, Masson, Akadémiai Kiadó, Paris, France, 1967, English translation North-Holland, Amsterdam, The Netherlands, and Akadémiai Kiado, Budapest, Hungary, 1970.

[27] P. D. Lax and R. S. Phillips, Scattering Theory, Academic Press, New York, NY, USA, 1967. 


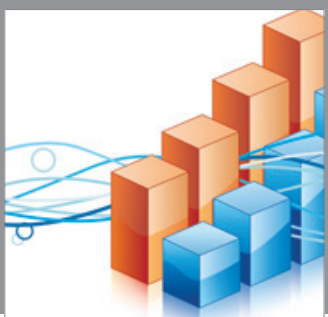

Advances in

Operations Research

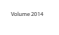

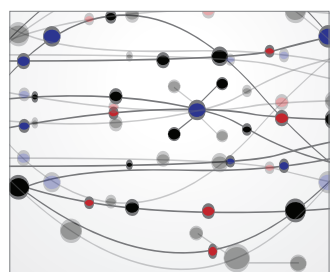

\section{The Scientific} World Journal
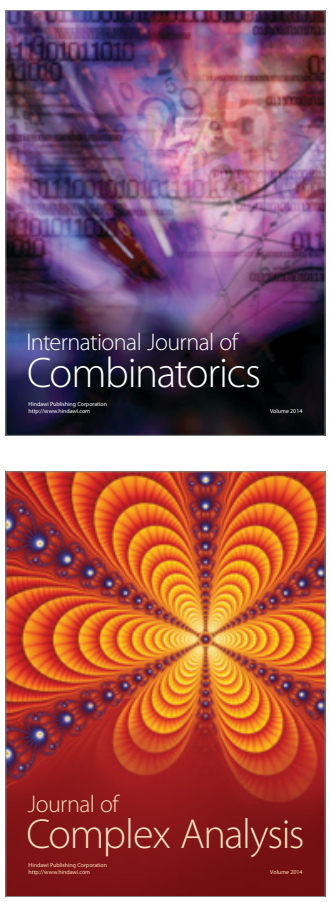

International Journal of

Mathematics and

Mathematical

Sciences
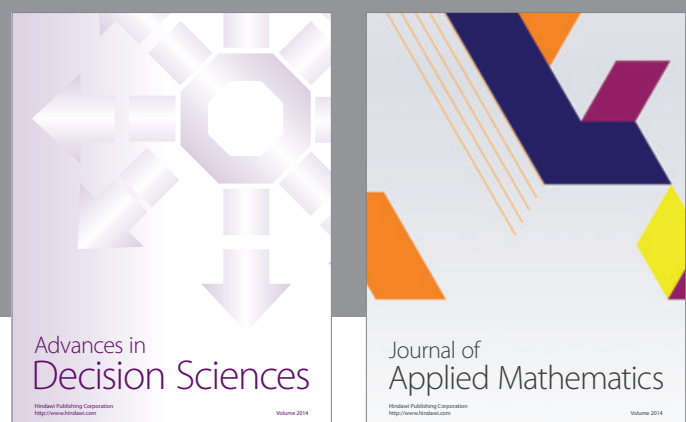

Journal of

Applied Mathematics
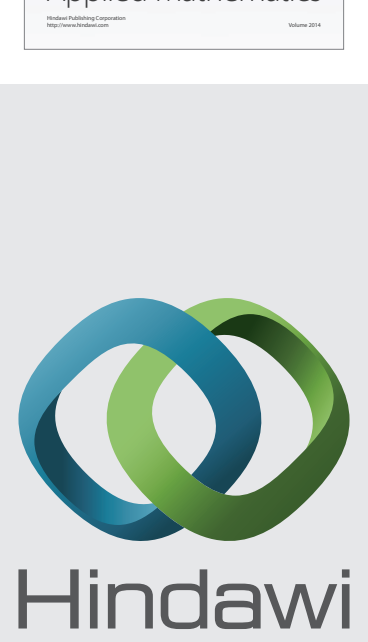

Submit your manuscripts at http://www.hindawi.com
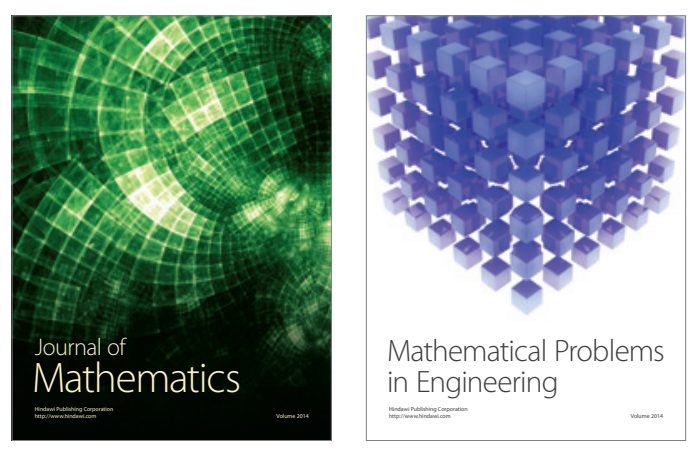

Mathematical Problems in Engineering
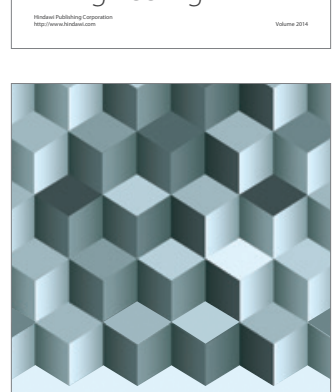

Journal of

Function Spaces
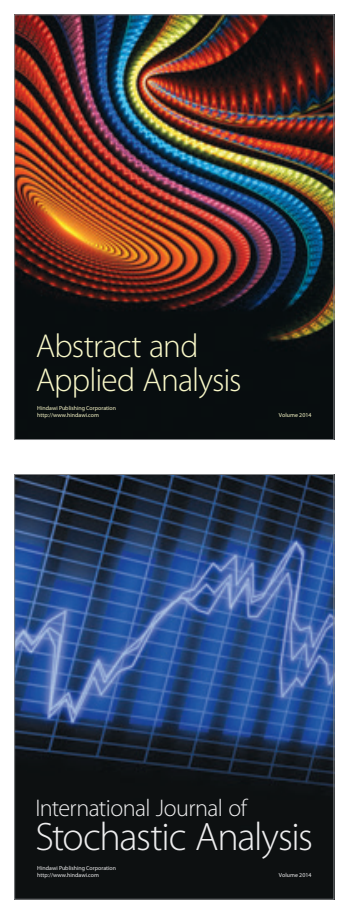

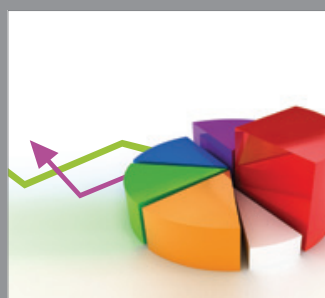

ournal of

Probability and Statistics

Promensencen
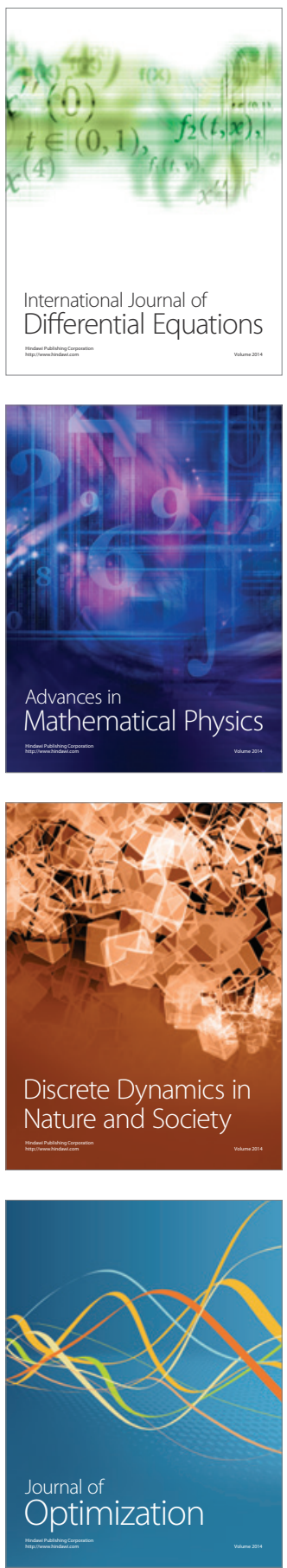